\title{
Clinical Management of Hypertriglyceridemia in the Prevention of Cardiovascular Disease and Pancreatitis
}

\author{
Patricia Hernandez ${ }^{1} \cdot$ Neena Passi $^{1} \cdot$ Taher Modarressi $^{2,3} \cdot$ Vivek Kulkarni $^{1} \cdot$ Meshal Soni $^{1} \cdot$ Fran Burke $^{1}$. \\ Archna Bajaj ${ }^{1} \cdot$ Daniel Soffer $^{1}$ (D)
}

Accepted: 24 July 2021 / Published online: 13 September 2021

(c) The Author(s), under exclusive licence to Springer Science+Business Media, LLC, part of Springer Nature 2021

\begin{abstract}
Purpose of Review Hypertriglyceridemia (HTG) is common and is a significant contributor to atherosclerosis and pancreatitis risk. Specific HTG treatments have had variable success in reducing atherosclerosis risk. Novel therapies for severe HTG treatment and pancreatitis risk reduction are likely to be available soon. These novel therapies are expected to have broader applications for more moderate HTG and atherosclerosis risk reduction as well.

Recent Findings NHANES 2012 data has confirmed a reduction in average triglyceride (TG) levels in the US population. Dietary modification and weight reduction when needed remain the core treatment elements for all individuals with HTG, while statin therapy is a foundational pharmacologic care for atherosclerotic cardiovascular disease (ASCVD) event risk reduction. In addition, the REDUCE-IT study provides evidence for additional benefit from the use of highdose icosapent ethyl (IPE) on top of background medical therapy in adults with moderate HTG and ASCVD or type 2 diabetes mellitus (T2D) and additional ASCVD risk factors. However, treatment with eicosapentaenoic acid (EPA) combined with docosahexanoic acid (DHA) did not reduce ASCVD in a similar population studied in the STRENGTH trial. Furthermore, novel therapeutics targeting PPAR-a, as well as ApoC-III and AngPTL3, effectively lower TG levels in individuals with moderate and severe HTG, respectively. These treatments may have applicability for reducing risk from ASCVD among individuals with chylomicronemia; in addition, ApoC-III and AngPTL3 treatments may have a role in treating individuals with the rare monogenic familial chylomicronemia syndrome (FCS) at risk for acute pancreatitis (AP).

Summary Residual ASCVD risk in individuals treated with contemporary care may be due in part to non-LDL lipid abnormalities including HTG. The findings from REDUCE-IT, but not STRENGTH, confirm that consumption of high-dose EPA may reduce ASCVD risk, while combination therapy of EPA plus DHA does not reduce ASCVD in a similar population. TG lowering likely reduces ASCVD risk in individuals with HTG, but ASCVD risk is multifactorial; the added benefit of IPE to contemporary preventive therapy is the consequence of differential non-TG biologic properties between the two fatty acids. Acute pancreatitis is more difficult to study prospectively since it is less common; however, TG lowering is likely critical for the care of at-risk individuals. Additional benefit from novel therapy that has an impact on this otherwise refractory condition is anticipated.
\end{abstract}

Keywords Hypertriglyceridemia $\cdot$ Triglyceride-rich lipoproteins $\cdot$ Atherosclerosis $\cdot$ Acute pancreatitis $\cdot$ Lipoprotein lipase

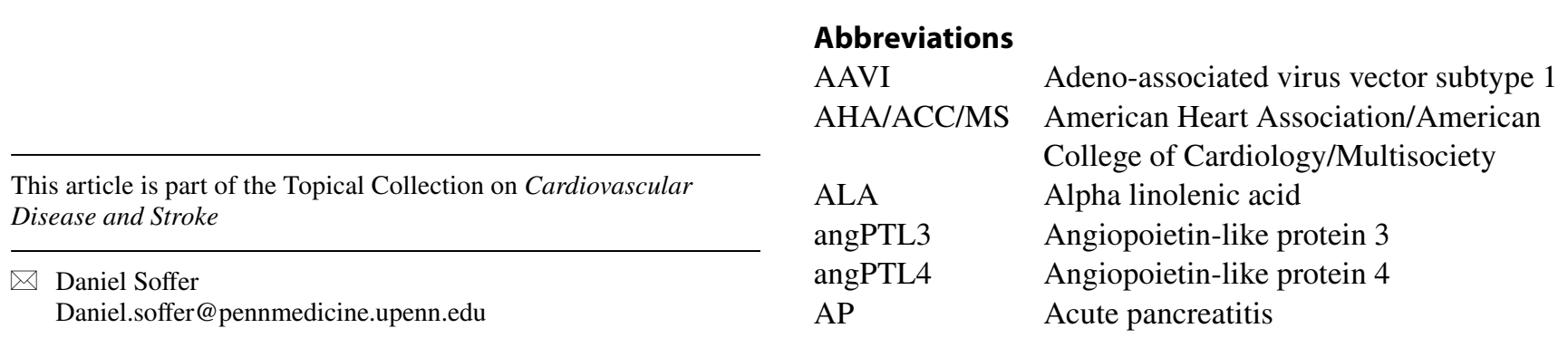

Extended author information available on the last page of the article 


\begin{tabular}{|c|c|}
\hline apoB & Apolipoprotein B \\
\hline apoC-III & Apolipoprotein C-III \\
\hline apoE & Apolipoprotein E \\
\hline ASCVD & Atherosclerotic cardiovascular disease \\
\hline ASO & Antisense oligonucleotide \\
\hline CETP & Cholesteryl ester transfer protein \\
\hline $\mathrm{CM}$ & Chylomicron \\
\hline $\mathrm{CMr}$ & Chylomicron remnant \\
\hline CVD & Cardiovascular disease \\
\hline DHA & Docosahexaenoic acid \\
\hline EPA & Eicosapentaenoic acid \\
\hline FA & Fatty acid \\
\hline FCHL & Familial combined hyperlipidemia \\
\hline FCS & Familial chylomicronemia syndrome \\
\hline FDBL & Familial dysbetalipoproteinemia \\
\hline FHTG & Familial hypertriglyceridemia \\
\hline $\mathrm{GH}$ & Growth hormone \\
\hline HL & Hepatic lipase \\
\hline hPGH & Human placental growth hormone \\
\hline hPL & Human placental lactogen \\
\hline hsL & Hormone-sensitive lipase \\
\hline HTG & Hypertriglyceridemia \\
\hline HTG-AP & $\begin{array}{l}\text { Hypertriglyceridemia-associated } \\
\text { pancreatitis }\end{array}$ \\
\hline IDL & Intermediate-density lipoprotein \\
\hline LA & Linoleic acid \\
\hline LD & Lipodystrophy \\
\hline LP & Lipoproteins \\
\hline LPL & Lipoprotein lipase \\
\hline MCS & $\begin{array}{l}\text { Multifactorial chylomicronemia } \\
\text { syndrome }\end{array}$ \\
\hline MCT & Medium chain triglyceride \\
\hline mRNA & Messenger ribonucleic acid \\
\hline MUFA & Monounsaturated fatty acid \\
\hline NAFLD & Non-alcoholic fatty liver disease \\
\hline NHANES & $\begin{array}{l}\text { National Health and Nutrition Examina- } \\
\text { tion Survey }\end{array}$ \\
\hline NLA & National Lipid Association \\
\hline O3FA & Omega-3 fatty acid \\
\hline PCSK9 & Proprotein convertase subtilisin/kexin-9 \\
\hline PPAR-a & $\begin{array}{l}\text { Peroxisome proliferator-activated } \\
\text { receptor- } a\end{array}$ \\
\hline PUFA & Polyunsaturated fatty acid \\
\hline SFA & Saturated fatty acid \\
\hline T2DM & Type 2 diabetes mellitus \\
\hline $\mathrm{TC}$ & Total cholesterol \\
\hline TG & Triglycerides \\
\hline TRL & Triglyceride-rich lipoprotein \\
\hline TRLr & Triglyceride rich lipoprotein remnant \\
\hline VLDL & Very low-density lipoprotein \\
\hline VLDLr & Very low-density lipoprotein remnant \\
\hline VLFD & Very low-fat diet \\
\hline
\end{tabular}

\section{Introduction}

Hypertriglyceridemia (HTG) is a common condition associated with multiple medical concerns. Most importantly, HTG increases the risk for atherosclerotic cardiovascular disease (ASCVD) and acute pancreatitis (AP), and national guidelines support attention to and treatment of HTG to reduce risk. We provide an update on the background, mechanisms, epidemiology, manifestations, interventions, and rationale for the treatment of HTG and review novel therapies in development.

\section{Summary of TG-Rich Lipoprotein Life Cycle}

Triglycerides (TG) are non-polar macromolecules with poor solubility in blood that require lipoproteins (LP) to circulate. There are multiple mechanisms that regulate the synthesis, condensation, and remodeling of TG-rich LP (TRL) and clearance from the circulation. Serum TG levels are heavily influenced by a combination of environmental and genetic factors. Environmental conditions that may influence TG levels include diet, alcohol intake, physical activity, pharmacology, adipose function, skeletal muscle function/activity, liver fat, insulin resistance or deficiency, and hormonal status. Rarely does the inheritance of biallelic genetic variants or the inheritance of multiple less severe variants impacts TG levels without significant environmental influences.

Since their inception, national and international lipid guidelines have been consistent about the need to manage severe HTG to prevent AP. However, guidelines have varied in recommendations to manage moderate HTG for the prevention of ASCVD as clinical data unfolds in this space [1]. Nonetheless, HTG management strategies uniformly emphasize the importance of a healthy lifestyle, especially dietary intervention and weight reduction when needed, and recent guidelines affirm a treatment strategy.

\section{Hypertriglyceridemia Diagnosis and Classification}

The major US and European lipid guidelines have designated different thresholds for HTG classification (Table 1).

HTG classification is based on fasting serum TG concentrations, but non-fasting serum TG concentrations may have improved predictive value for ASCVD. The European guideline supports a non-fasting lipid profile, and the 2018 AHA/ACC/MS guideline recommends either fasting or nonfasting lipid profile for screening and follow-up fasting lipid profile if serum TG concentrations are $>400 \mathrm{mg} / \mathrm{dL}[2,3]$. In the absence of uniform global recommendations, fasting TG may be favored for more precise diagnosis and classification 
of HTG, but non-fasting serum TG concentrations are preferred to improve patient satisfaction.

Observational cohort studies suggest that HTG-associated acute pancreatitis (HTG-AP) can occur with any elevation in serum TG concentrations, but the risk increases proportional to the TG level. Accordingly, the AHA/ACC/MS guideline designates serum TG concentrations of $\geq 500 \mathrm{mg} / \mathrm{dL}$ as severe HTG [2]. While the risk for AP does increase when there is chylomicronemia, HTG due to very low-density lipoprotein (VLDL)-TG excess may also occur, and the absence of chylomicronemia should not dissuade attention for AP risk mitigation.

\section{Epidemiology: Prevalence, Gender, and Ethnic Differences}

The National Health and Nutrition Examination Survey (NHANES, 2001-2012, n > 4000) estimated that approximately $30 \%$ of US adults have HTG $(\geq 150 \mathrm{mg} / \mathrm{dL})$ and $1.7 \%$ have severe HTG ( $\geq 500 \mathrm{mg} / \mathrm{dL})$ [5]. Fortunately, there has been an observed decline in the prevalence of HTG from $33.3 \%$ in the 2001-2004 survey to $25.1 \%$ in the 2009-2012 survey [5]. Severe HTG $(\geq 880 \mathrm{mg} / \mathrm{dL}[10 \mathrm{mmol} / \mathrm{L}])$ was found in $<0.1 \%$ of the Danish population in the Copenhagen City Heart Study, and an electronic medical record review of a Dallas County health system, with a proportionally higher than national average Hispanic population, found that $<0.14 \%$ had at least one TG value $\geq 2000 \mathrm{mg} / \mathrm{dL}$ $(22.6 \mathrm{mmol} / \mathrm{L})[6,7]$.

The overall prevalence of HTG is higher in men than in women ( $28.7 \%$ and $21.5 \%$, respectively), with the highest prevalence in the 40- to 59-year age group in men and in the over 60-year age group in women. The prevalence of HTG is increasing among youth and adolescents and is attributed to increasing rates of obesity and diabetes. Mexican-Americans have nearly twice the prevalence of HTG as non-Hispanic Black Americans (34.9\% vs $15.6 \%$ ) [5].

Rates of HTG in the European population are comparable to that of the USA. The DECODE study, based on analysis of nine European population cohorts in the 1990s, found the prevalence of HTG to be $36.4 \%$ in men and $24.8 \%$ in women [8]. Large population databases with detailed lipid/LP data are lacking from most of Africa and much of Asia. As a result, the medical community is missing out on important opportunities to better understand the impact of genetics and environmental effects on TG metabolism.

\section{Hypertriglyceridemia Causes}

Serum TG levels are influenced by a number of environmental and genetic factors. Individuals with persistent HTG can have monogenic, polygenic, and unknown genetic causes. Secondary contributors include diet, alcohol intake, activity level, medications, and metabolic states. Inherited and environmental factors may cause HTG by enhancing TG synthesis, increasing production of TRL, and inhibiting peripheral TG lipolysis and/or clearance of TRL.

\section{Obesity, Diabetes/Insulin Resistance, and Lipodystrophy}

The pathophysiology of HTG in several overlapping metabolic conditions such as obesity, type 2 diabetes (T2DM), metabolic syndrome, and lipodystrophies is multifactorial. Each of these conditions has a spectrum of manifestations and is influenced by numerous genetic and environmental factors. As such, individuals with apparently similar clinical characteristics may have divergent manifestations.
Table 1 Classification of $\mathrm{H} \backslash$ hypertriglyceridemia

\begin{tabular}{lll}
\hline Society & Category & $\begin{array}{l}\text { Serum triglyceride } \\
\text { concentration mg/dL } \\
(\mathrm{mmol} / \mathrm{L})\end{array}$ \\
\hline $\begin{array}{c}\text { American Heart Association/American College of } \\
\text { Cardiology/Multisociety [2] }\end{array}$ & $\begin{array}{l}\text { Normal } \\
\text { Moderate }\end{array}$ & $\begin{array}{c}\leq 175(\leq 2.0) \\
175-499(2.0-5.6)\end{array}$ \\
European Society of Cardiology [3] & Severe & $\geq 500(\geq 5.7)$ \\
& Normal & $<150(<1.7)$ \\
Endocrine Society [4] & Mild-moderate & $150-880(1.7-9.9)$ \\
& Severe & $>880(>10)$ \\
& Normal & $<150(<1.7)$ \\
& Mild & $150-199(1.7-2.3)$ \\
& Moderate & $200-999(2.3-11.2)$ \\
& Severe & $1000-1999(11.2-22.4)$ \\
& Very severe & $\geq 2000(>22.4)$ \\
\hline
\end{tabular}

Major guidelines classifying level of hypertriglyceridemia based upon specific thresholds 
Lipodystrophies (LD) are a heterogenous group of both genetic and acquired conditions characterized by lipoatrophy and metabolic abnormalities. Insulin resistance, dyslipidemia, non-alcoholic fatty liver disease (NAFLD), and leptin deficiency predominately underlie all forms $[9,10]$. HTG may be mild, moderate, or severe in individuals with LD and should be considered when evaluating patients with appropriate phenotype and body morphology.

\section{Organopathies}

Various hepatic diseases, especially NAFLD, are associated with HTG. This is thought to be due to increases in hepatic lipogenesis associated with insulin resistance $[11,12]$. Separately, polymorphisms in APOC3 associated with HTG are correlated with NAFLD [13].

Nephrotic syndrome and kidney failure increase hepatic VLDL production and/or decrease clearance due to downregulation of LPL and hepatic lipase (HL) activity [14]. The role of angiopoietin-like 4 (ANGPTL4) upregulation in nephrotic syndrome may have a significant role as well [15].

\section{Endocrinopathies}

Endocrinopathies are associated with HTG, including hypothyroidism, Cushing's syndrome, acromegaly, male hypogonadism, hyperprolactinemia, and polycystic ovary syndrome, as summarized in Table 2 . In addition, many of these conditions lead to metabolic diseases that independently worsen HTG.

\section{Pregnancy}

Pregnancy is characterized by multiple adaptations in lipid metabolism that ensure normal maternal and fetal energy needs are met [35]. Several factors potentiate HTG, which in healthy women can rise up to 3 times higher than baseline levels by the third trimester [36]. Rising concentrations of placental hormones such as human placental lactogen (hPL) and human placental growth hormone (hPGH) increase insulin resistance, while rising estrogen stimulates hepatic VLDL production and suppresses lipase activity [37, 38].

Severe HTG-associated acute pancreatitis (HTG-AP) can occur in second and third trimesters of pregnancy in women with diabetes, insulin resistance, obesity, and prenatal moderate HTG [39]. Identification of and counseling for women at risk for HTG prior to pregnancy are critical [40, 41]. Dietary modification, pharmacotherapy, and plasmapheresis are available treatment modalities, and all have specific risks and benefits in this high-risk condition. There is emerging data about the relationship between maternal HTG with fetal adiposity and whether this increases obesity risk in long term [42].

\section{Medications that Raise TG}

Many medications raise TG as a side effect, particularly in people with underlying inherited predisposition [43]. The more commonly used medicines include thiazide diuretics, beta-blockers, bile acid resins, specific immune-modulating therapies, protease inhibitors, retinoids, atypical antipsychotics, glucocorticoids, estrogens, and selective estrogen receptor modulators. Oral estrogen, but not topical (e.g. patches, vaginal cream), either as oral contraceptives or post-menopausal hormone replacement can exacerbate HTG $[44,45]$.

\section{Genetic Hypertriglyceridemia}

Moderate and severe HTG syndromes are the result of polygenic inheritance of recessive genes and the combination with strong environmental influences. The most severe manifestations of inherited HTG, which are caused by monogenic

Table 2 Endocrinopathies and mechanism for causing hypertriglyceridemia

\begin{tabular}{ll}
\hline Disorder & Mechanism \\
\hline Hypothyroidism [16] & $\begin{array}{l}\text { Decreased clearance via HL/LPL [17] } \\
\text { Increased AngPTL3 expression [18] } \\
\text { Note: not observed in subclinical disease [19, 20] } \\
\text { Insulin resistance, changes in FFA metabolism and adipocyte function, typically accompanied by diabetes and } \\
\text { obesity [22] } \\
\text { Growth hormone (GH) direct inhibitory inhibits HL/LPL accompanied by insulin resistance and metabolic } \\
\text { Acromegaly [23] } \\
\text { diseases [24, 25] } \\
\text { Male hypogonadism [26] }\end{array} \quad \begin{array}{c}\text { Proposed mechanisms include hypothalamic-pituitary-gonadal axis disruption, weight changes and subsequent } \\
\text { Hyperprolactinemia [29] }\end{array}$ \\
insulin resistance, or possibly direct LPL effects [30-32] \\
Polycystic ovary syndrome [33] & Insulin resistance, hyperandrogenism, often exacerbated by obesity and/or diabetes [34]
\end{tabular}

List of endocrinopathies that contributes to hypertriglyceridemia and proposed mechanisms 
biallelic transmission, are rare; as such, individuals tend not to have severely affected relatives.

The National Lipid Association (NLA) Scientific Statement on Genetic Testing in Dyslipidemia rates genetic testing for HTG as class III (may be harmful) or IIb (reasonable to perform) if familial chylomicronemia syndrome (FCS) is suspected [46•]. In addition, the European Working Group on FCS has created a clinical tool with high sensitivity and specificity for distinguishing FCS from the more common multifactorial chylomicronemia syndrome (MCS) without the use of genetic testing [47].

\section{Monogenic}

\section{Familial Dysbetalipoproteinemia (FDBL)}

FDBL is characterized by excess levels of circulating TRL remnant particles (CM remnant [CMr], VLDL remnant [VLDLr], IDL) due to impaired clearance as a result of functional defect in apolipoprotein E (apoE) [48]. Biallelic common (APOE2/E2) and rare genetic variants in APOE result in dysbetalipoproteinemia (increased proportion of TRL remnants), though hyperlipidemia and pathologic phenotype (previously described as Fredrickson hyperlipoproteinemia type III) manifest only after a secondary environmental challenge occurs (commonly referred to as a "second hit") such as weight gain, dietary challenge, insulin resistance/ diabetes mellitus, hormonal changes, or pharmacotherapy [49]. FDBL is suspected in individuals who develop a severe increase in background cholesterol and TG levels, have combined hyperlipidemia with TC approximately equal to TG levels, and have normal apoB indicating highly cholesterolenriched LP [50]. In addition to hyperlipidemia, eruptive, tuberous, and palmar crease xanthomas may be present, and there is an increased association with ASCVD, especially peripheral arterial disease.

FDBL treatment includes focused attention on management of secondary environmental factors and pharmacotherapy with high-dose omega-3 fatty acids (O3FA), fibrates, and/or statins.

\section{Familial Chylomicronemia Syndrome (FCS)}

FCS is a rare monogenic condition that occurs in roughly one in 1 million in the general population. It is mediated by biallelic genetic variants in lipoprotein lipase $(L P L)$ or variants in LPL cofactors (e.g., APOA5, APOC2, LMF1, $G P 1 H B P 1)$ in their homozygous, compound, or double heterozygous forms, though specific genetic variants are not always identified [47, 51, 52]. FCS is characterized by low or no LPL activity that results in persistent, severe HTG due to chylomicronemia and high risk for AP.
FCS normally presents in childhood or at a later age precipitated by pregnancy or estrogen-containing oral contraceptives. It is not otherwise accompanied by secondary environmental factors (e.g., obesity, diabetes, hypothyroidism). Children may present as early as infancy with failureto-thrive, steatorrhea, and/or eruptive xanthoma, and adults tend to be under or normal weight.

Patients with FCS tend to experience persistently elevated TG levels $>880 \mathrm{mg} / \mathrm{dL}(10 \mathrm{mmol} / \mathrm{L})$, which require prolonged fasting followed by a very low-fat diet (VLFD) to clear chylomicrons (CM). FCS is associated with low apoB, high TG:apoB ratio $>10.5(\mathrm{mg} / \mathrm{dL})$, and a TG:TC ratio $>5: 1$ $(\mathrm{mg} / \mathrm{dL})$. Because CM do not directly contribute to atherosclerosis, premature ASCVD is not expected. However, accompanying ASCVD risk factors and VLDL excess may promote ASCVD in patients with FCS later in life.

\section{Polygenic}

\section{Multifactorial Chylomicronemia Syndrome (MCS)}

MCS is characterized by chylomicronemia and severe HTG. MCS differs from FCS in the variability of LPL activity, TG variability, and obligatory presence of environmental factors. Individuals with MCS have either polygenic or unknown genetic causes for LPL dysfunction, and one or multiple environmental factors are present. MCS is commonly associated with obesity, insulin resistance, and diabetes mellitus. Compared to FCS, MCS is also more likely to respond to pharmacotherapy and has a higher population frequency ( $>20$ times more than FCS) [47]. Of note, in the previously mentioned Dallas County medical chart review by Esparza et al., 103 patients out of approximately 70,000 had TG levels $>2000 \mathrm{mg} / \mathrm{dL}$; of those, only 2 had classic FCS, while the rest $(n=101)$ had characteristics of MCS [7]. There is high risk for both AP and ASCVD in patients with MCS.

The diagnosis of FCS is important to distinguish from MCS because HTG due to FCS tends not to respond to contemporary FDA-approved pharmacotherapy. When FCS is confirmed, dietary modification is the principal therapeutic modality. Primary treatment of MCS includes management of secondary environmental factors especially focusing on dietary modification, physical activity, weight reduction when needed, glycemic control, and liberal use of pharmacotherapy for TG-reduction (e.g., fibric acid derivatives, high-dose omega-3 fatty acids [O3FA], and statin therapy).

Familial hypertriglyceridemia (FHTG) is characterized in an individual who has persistent HTG due to VLDL excess without chylomicronemia (previously described as type IV Fredrickson phenotype) or TRL remnants, variable ASCVD risk, and a history of other family members with the same. These patients have moderate 
to severe HTG due to persistent VLDL-TG elevations, normal apoB, and TG:TC ratio approximately 5:1. The specific phenotype may change, and patients may develop chylomicronemia if exposed to significant environmental challenges. There may be an increased risk of ASCVD and AP, but much lower risk for ASCVD than familial combined hyperlipidemia (FCHL) described below, and lower risk for AP than the chylomicronemia syndromes. There are multiple genetic variants associated with this condition, but individuals with this pattern tend to have other family members with the same lipid/lipoprotein phenotype, and a variety of genotypes have been described. Enhanced TG synthesis in individuals with FHTG is observed [53]. Treatment should be based upon assessment of AP risk due to HTG and for ASCVD risk management according to the US AHA/ACC/MS cholesterol guideline [2].

Familial combined hyperlipidemia (FCHL) is diagnosed in individuals who have persistent combined hypercholesterolemia and moderate to severe HTG, a family history of premature ASCVD and variable family history of lipid abnormalities, a personal history of elevated apolipoprotein B (apoB), and exclusion of autosomal dominant monogenic familial hypercholesterolemia (FH) [54]. Overproduction of apoB may be accompanied by overproduction of VLDL and impaired clearance of LDL, VLDL, and TRL [55]. Patients with FCHL have higher ASCVD risk than individuals with heterozygous familial hypercholesterolemia, and high-intensity statin should be prescribed. Additional non-statin treatment should be considered to optimize non-HDL-C/apoB reduction matched to the severity of ASCVD risk, in accordance with guideline- and evidencebased approaches.

\section{Medical Consequences of Hypertriglyceridemia}

TG levels will affect plasma appearance due to the ability of larger TRL (VLDL and CM) to disrupt light transmission. When TG levels are normal, the predominant apoB-containing LP in circulation is low-density lipoprotein (LDL), and the plasma is transparent. When TG $>150 \mathrm{mg} / \mathrm{dL}$ (VLDL and/or TRL remnant concentrations are elevated), plasma may be opalescent. When TG $>500 \mathrm{mg} / \mathrm{dL}, \mathrm{CM}$ are present, and the plasma will tend to be opaque or appear "lipemic" or milky [Fig. 1].

HTG may be associated with a variety of signs and symptoms including depression, memory loss, fatigue, back or abdominal pain, nausea, bowel dysfunction, neuropathy, and others. However, the most significant consequences of HTG are ASCVD and AP.

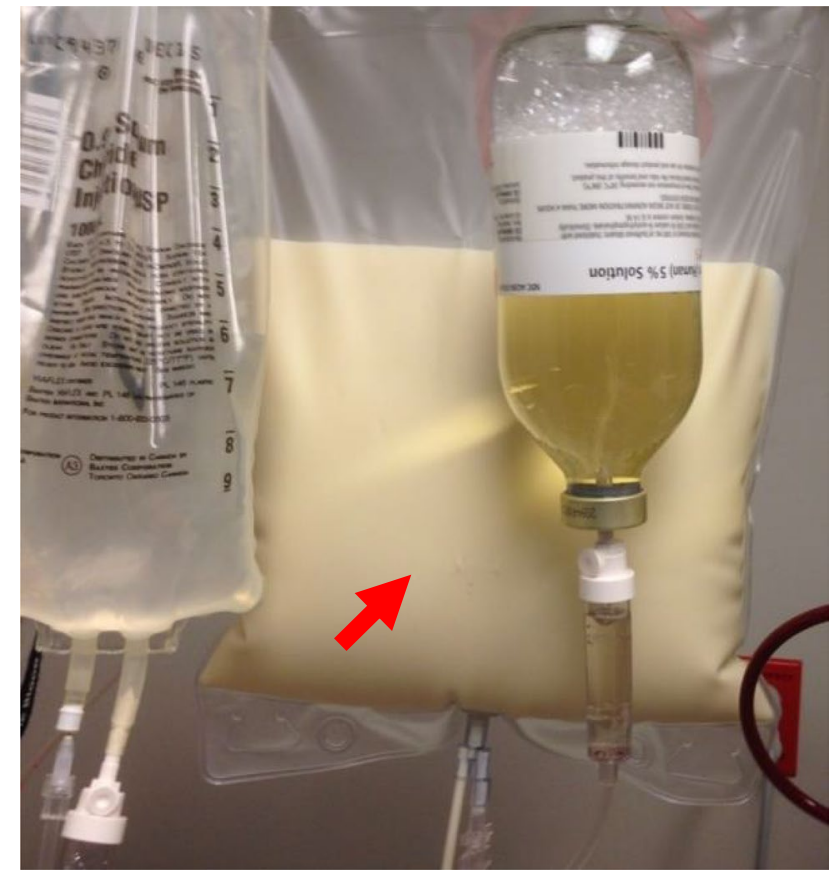

Fig. 1 (Chylous serum). Image of milky plasma in waste bag collected during plasma exchange in patient with hyperchylomicronemia (shown with permission from patient [on file])

\section{Atherosclerotic Cardiovascular Disease (ASCVD)}

Mendelian randomization studies support a causative impact of TG-associated genetic loci on ASCVD risk [56, 57]. HTG is independently associated with ASCVD risk in multivariate observational analyses as well. TRL and TRL remnant particles are directly atherogenic, and HTG also promotes atherogenesis indirectly because of localized FFA inflammatory impact on endothelial function. Additionally, because HTG is highly associated with obesity, insulin resistance/ diabetes, hypertension, and other major ASCVD risk factors, there is also a bystander (or association) effect.

An example of the combined and complex interaction between HTG and comorbidities is seen in a meta-analysis of 31 studies with a total of 132, 044 patients with T2DM. A subgroup analysis adjusting for blood pressure and glycemic levels confirmed a consistent association between TG levels and cardiovascular disease (CVD) risk for T2DM patients [49, 50]. Furthermore, additional subgroup analyses examining the incidence of different types of CVD found an association between HTG and risk for coronary heart disease but not stroke in this diabetic cohort [58]. A different retrospective, longitudinal study examined the association of HTG and ASCVD in a cohort of 158, 042 low to moderate risk participants with a calculated 10-year ASCVD risk of approximately $7 \%$. When compared to study participants with normal TG levels, participants with TG $150-500 \mathrm{mg} /$ $\mathrm{dL}$ demonstrated a 1.6-fold increased risk of future ASCVD 
events [59]. These study findings emphasize the significance of HTG even in a low to moderate risk population for the risk assessment of future ASCVD and support the guideline recommendations to consider HTG as a risk-enhancing factor.

\section{Acute Pancreatitis (AP)}

HTG is associated with proportional risk for developing acute pancreatitis (AP), and HTG-AP accounts for approximately $10 \%$ of AP cases; only gallstone disease and alcohol are more common causes of AP. HTG cohort studies have demonstrated that the prevalence of HTG-AP in individuals with TG> $>1000 \mathrm{mg} /$ $\mathrm{dL}$ is as high as approximately $20 \%$. Murphy et al. demonstrated that in a stable Scottish population, even moderate HTG is associated with AP [60]. Gaudet et al. reported $>15 \times$ odds ratio for developing AP in individuals with TG levels $5-9 \mathrm{mmol} / \mathrm{L}$ $(\sim 442-796 \mathrm{mg} / \mathrm{dL}),>50 \times$ in individuals with $\mathrm{TG}>9 \mathrm{mmol} / \mathrm{L}$ $(796 \mathrm{mg} / \mathrm{dL})$, and $>300 \times$ in those with $\mathrm{TG}>9 \mathrm{mmol} / \mathrm{L}$ and biallelic LPL mutations in a pattern consistent with FCS [61, 62]. HTG-AP may be more severe than other causes and may be particularly high risk during pregnancy [63].

Mechanistically, it has been proposed that CM excess is needed to provoke HTG-AP. In fact, Gonzales et al. demonstrated that a marker of $\mathrm{CM}$ persistence (TG/apoB > 10.6) has a high sensitivity and specificity for identification of HTG-AP risk but does not identify a specific threshold for the potential occurrence of AP [64].

\section{Other Manifestations}

Severe HTG can lead to dermatologic, hematologic, and ophthalmologic stigmata as well. Eruptive xanthomas, 1-5 mm orange-yellow papules often pruritic, may be associated with chylomicronemia and are found on extensor surfaces including the elbows, shoulders, and buttocks [Fig. 2]. Tuberous xanthomas are firm, non-tender, red-yellow nodules containing foam cells. They are found on body surfaces prone to pressure including the knees, heels, and elbows and may be seen in familial dysbetalipoproteinemia (FDBL) [65, 66]. Yellow-orange palmar creases known as xanthoma striata palmaris may also be seen and are pathognomonic for FDBL. Cream-colored opacification of the retinal blood vessels (known as lipemia retinalis) due to hyperchylomicronemia may be visualized during fundoscopic examination.

In addition to HTG-AP, individuals with FCS report abdominal pain without the diagnosis of AP as a common complaint, as well as other non-specific gastrointestinal, neurocognitive, peripheral neuropathy, depressed mood, and other effects [67].

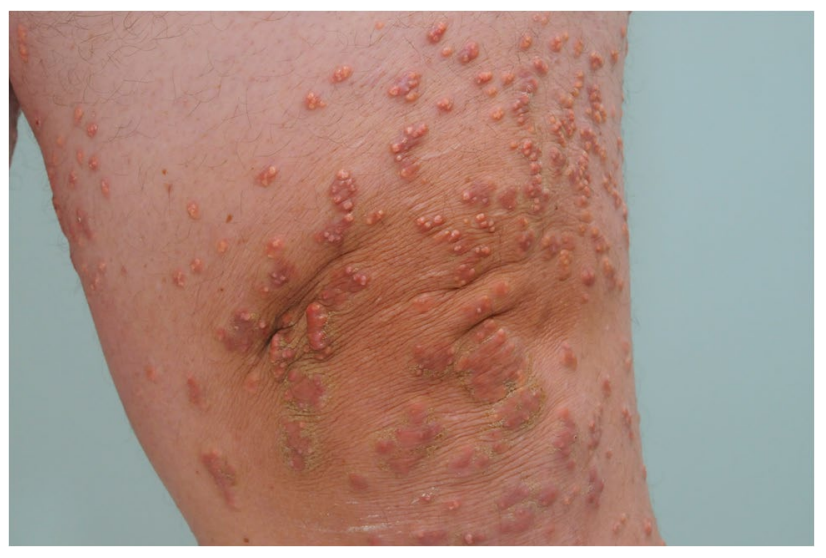

Fig. 2 Eruptive xanthomas. Eruptive xanthomas associated with hyperchylomicronemia (image shared with patient's permission [on file])

\section{Dietary Management of Hypertriglyceridemia}

\section{Moderate Hypertriglyceridemia}

Body weight and fat distribution, especially visceral adiposity, affect serum TG levels. HTG has been noted in $>80 \%$ of overweight or obese individuals $[68,69]$. Weight loss can enable TG reductions proportional to baseline TG levels and amount of weight loss achieved. It has been shown that a 5 to $10 \%$ weight reduction lowers TG by approximately $20 \%$ $[68,70]$.

Although optimal dietary macronutrient content may be flexible, decreased intake of carbohydrates while maintaining a healthy body weight has been shown to be beneficial in TG lowering in patients with borderline high to high levels $<500 \mathrm{mg} / \mathrm{dL}$ [71]. A meta-analysis of 60 controlled trials showed that a $1 \%$ decrease in calories from carbohydrates replaced with an isocaloric increase in fat reduces fasting TG levels by 1 to $2 \%$ [72]. Either polyunsaturated fat (PUFA) and/or monounsaturated fat (MUFA) is the preferred replacement nutrient rather than saturated fat (SFA).

The adverse effects of a high-carbohydrate diet on TG occur mainly when refined sources of carbohydrate, including table sugar, raw sugar, honey, and agave nectar, are consumed in excess [3]. Most sugars, because of fructose content, stimulate hepatic FA synthesis and increase hepatic fat accumulation, promoting VLDL production [73]. Fructose is present as high fructose corn syrup in many sugar-sweetened beverages ( $55 \%$ fructose, $45 \%$ glucose), which are the leading sources of added sugars in the American diet [73]. Whole fruit consumption as an alternative to fruit juice is recommended, and water consumption is preferred. Patients should be instructed to substitute fiber-rich whole grains and 
legumes for refined starches; however, quantity and calories still need to be considered [71].

\section{Severe Hypertriglyceridemia}

Patients with severe HTG require a VLFD to clear CM from circulation and reduce AP risk. Dietary fat should be limited to $<15 \%$ of total calories or $<20-30 \mathrm{~g}$ per day, since CM are derived from intestinal fat. Sources of both saturated and unsaturated fat should be reduced along with refined carbohydrates. Once CM are cleared and TG levels return to $<500 \mathrm{mg} / \mathrm{dL}$ in an individual who does not have FCS, fat intake (MUFA and PUFA) can be liberalized, and nutrition therapy should focus on weight loss, restoration of insulin sensitivity, and improvement in glycemic control [71]. Alternative dietary strategies for weight reduction that do not limit fat consumption when there is severe HTG are not recommended because of AP risk [74]. See Fig. 3 for manifestations of severe hypertriglyceridemia.

\section{Familial Chylomicronemia Syndrome (FCS)}

FCS requires life-long adherence to VLFD containing $<15$ to $20 \mathrm{~g}$ of fat per day and limited intake of refined carbohydrates. Strict dietary adherence is challenging in the long term, and individuals with FCS report this as significantly impacting their quality of life. Medium chain TG (MCT) oils can be used to improve satiety and provide additional calories since they are not incorporated into CM, but are hydrolyzed to albumin, and transported directly to the liver via the portal vein. To meet essential FA requirements, $2-4 \%$ of total calorie intake from the essential FA, linoleic acid (LA), and $\alpha$-linolenic acid (ALA) is recommended, and fatsoluble vitamins should be supplemented as needed. Abstinence from alcohol is strongly advised [75]. Recommendations have been summarized in a simple one-page patient handout by the NLA, which can be a useful counseling tool [76].

\section{Pharmacotherapy for Hypertriglyceridemia}

Clinicians should identify and treat secondary causes, address lifestyle and dietary strategies, and prescribe HTG pharmacotherapy when needed. Fibric acid derivatives (fenofibrate instead of gemfibrozil if statin use is considered) and/or high-dose prescription omega- 3 fatty acid supplements (O3FA) are recommended as first-line pharmacotherapy options for severe HTG to achieve the primary goal of lowering TG levels to $<500 \mathrm{mg} / \mathrm{dL}$ and reducing the risk for AP. Statin drugs may reduce TG further, and niacin has limited use in contemporary care and should only be considered in individuals with severe HTG where the benefit of additional TG lowering offsets niacin's potential negative effects. Once TG lowering has been achieved and/ or in individuals with moderate HTG $(150-500 \mathrm{mg} / \mathrm{dL}$ or even $501-1000 \mathrm{mg} / \mathrm{dL}$ ), attention is refocused to managing increased ASCVD risk; statins are foundational pharmacologic care for ASCVD risk reduction. According to the 2018 AHA/ACC/MS Cholesterol Guideline and 2020 NLA Position Statement, the use of ezetimibe, proprotein convertase subtilisin/kexin 9 (PCSK9) monoclonal antibodies,
Fig. 3 Manifestations of severe hypertriglyceridemia ( adapted from Davidson $\mathrm{M}$ et al. [61])

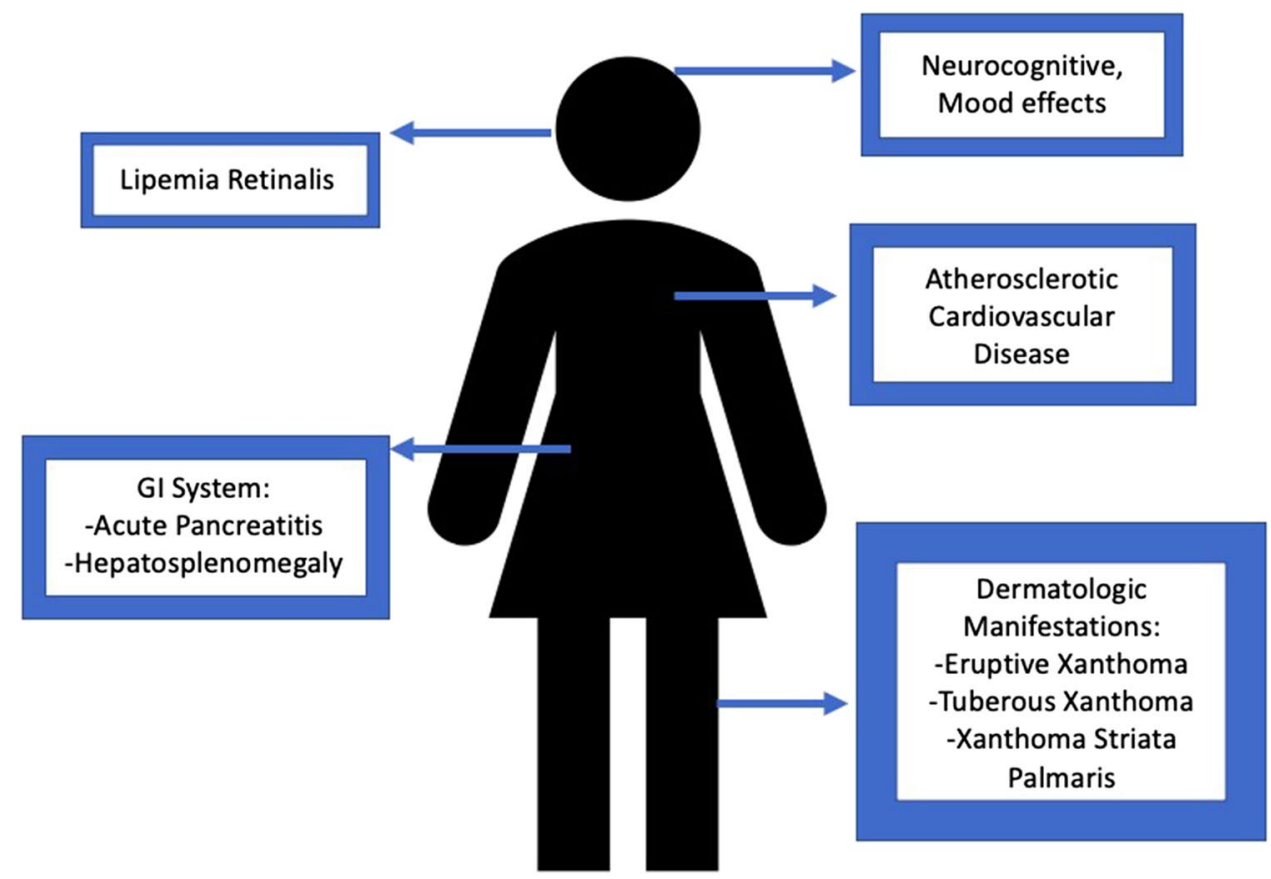


and icosapent ethyl should be considered in adults with clinical ASCVD who need additional lipid lowering to achieve ASCVD risk reduction [2, 77].

Additional pharmacotherapy for severe refractory HTG includes off-label use of orlistat (an intestinal lipase inhibitor) and lomitapide (microsomal TG transfer protein inhibitor). There are also investigational therapies that are being developed (discussed below).

\section{Omega-3 Fatty Acids (03FA)}

O3FA contain the long-chain PUFA, docosahexaenoic acid (DHA), and/or eicosapentaenoic acid (EPA) [78]. At doses of 3-4 g/daily, O3FA products reduce plasma TG by $25-50 \%$ by reducing hepatic production of VLDL as well as increasing VLDL clearance [79, 80]. Randomized controlled trials (RCT) evaluated the impact of O3FA on long-term cardiovascular (CV) disease outcomes with varying results. The Reduction of Cardiovascular Events with Icosapent EthylIntervention Trial (REDUCE-IT) compared icosapent ethyl (IPE), a purified EPA-only product, at a dose of $2 \mathrm{~g}$ twice daily with food, versus placebo as add-on to statin therapy in 8,175 adults with a history of CVD or with diabetes and at least one CVD risk factor, with TG levels between 150 and $500 \mathrm{mg} / \mathrm{dL}+/-10 \%[81 \bullet \bullet$ ]. After a median follow-up of 4.9 years, there was a $25 \%$ primary endpoint risk reduction for the treatment vs placebo groups. The Outcomes Study to Assess Statin Residual Risk Reduction with Epanova in High CV Risk Patients with Hypertriglyceridemia (STRENGTH) study was closed early in 2020 for futility in its attempt to show an effect of a high-dose EPA + DHA formulation of O3FA that can be taken without food, on CV

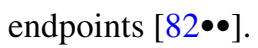

EPA and DHA have different biological properties, though both have TG-lowering effects. There have been many public discussions about the differential outcomes in REDUCE-IT vs STRENGTH; consideration of EPA dose and systemic EPA level achieved is chief among the most compelling conclusions about differential benefits. Some have argued that the difference in outcomes is due to different placebo comparisons (mineral oil vs corn oil, respectively). However, the FDA studied the effects of mineral oil and did not find reason to be concerned about its use as a placebo in REDUCE-IT [81••].

Until more is known, it is reasonable that any effective TG lowering achieved is worthwhile for AP risk reduction. However, high-dose IPE is favored over other O3FA preparations for reducing ASCVD risk in at-risk individuals with moderate HTG based upon differential RCT results.

\section{Fibrates}

Fibric acid derivatives lower TG levels by $25-50 \%$ by activating transcription factors for the nuclear peroxisome proliferator-activated a-receptors (PPAR-a) [83]. Fibrates reduce hepatic apoC-III production and increase LPL-mediated lipolysis. Fenofibrate and gemfibrozil are the two currently available fibrates in the USA. Gemfibrozil should not be used in combination with statins because of increased myopathy risk, but gemfibrozil does have evidence for ASCVD risk reduction as monotherapy in high-risk hyperlipidemia patients in the pre- or early statin era [84-86]. Fibrate therapy added to statin drugs has had disappointing ASCVD risk reduction in several RCT. However, post-hoc analysis of fibrate mono- and combination therapy RCT has demonstrated a consistent ASCVD risk reduction proportional to TG lowering in study participants with HTG and/ or low high-density lipoprotein cholesterol (HDL-C) [87].

Pemafibrate, which is in clinical development, is a selective PPAR-a agonist with greater efficacy than earlier generation fibrates. In phase 2 placebo-controlled trials, pemafibrate was shown to be associated with a $50 \%$ reduction in fasting plasma TG levels when combined with statin therapy in patients with baseline fasting TG levels $>200 \mathrm{mg} / \mathrm{dL}$ [88]. A phase 3 trial of pemafibrate (PROMINENT) is currently underway to assess long-term cardiovascular outcomes in approximately 10,000 participants with TG values between 200 and $500 \mathrm{mg} / \mathrm{dL}$ and T2DM [89].

\section{Gene Therapy for Familial Lipoprotein Lipase Deficiency}

Alipogene tiparvovec (Glybera) is an adeno-associated virus vector subtype 1 (AAV1) carrying the $L P L$ gain-of-function variant. The drug was approved in the European Union in 2012, but despite effective TG and AP risk lowering, it is no longer commercially available due to high cost and rare prescription [90, 91].

\section{Investigational Therapies}

Volanesorsen is an antisense oligonucleotide (ASO) that interferes with synthesis of apoC-III by selective inhibition of the APOC 3 mRNA. In a phase 3 clinical trial, once weekly injections of volanesorsen resulted in a $77 \%$ decrease in mean TG levels at 3 months in 33 patients with FCS [92]. Volanesorsen was approved in the European Union in 2019 for treatment of patients with FCS and is pending FDA review presently in the USA.

There are two angiopoietin-like 3 (ANGPTL3) inhibitor strategies in clinical development. A dose-ranging study of an ASO targeting ANGPTL3 mRNA (Vupanorsen) in 105 patients with fasting TG levels $>150 \mathrm{mg} / \mathrm{dL}, \mathrm{T} 2 \mathrm{DM}$, and hepatic 
steatosis showed a mean reduction in TG levels of 36-53\% after 6 months of treatment in the treated groups [93]. A second dose-ranging study of vupanorsen (TRANSLATE-TIMI 70) is ongoing and has enrolled patients with elevated TG and non-HDL cholesterol levels who are on a stable dose of statin [94]. Evinacumab is a human monoclonal antibody targeting ANGPTL3 that is further along in clinical development for HTG; it was approved in 2021 by the FDA for patients with homozygous familial hypercholesterolemia. Results from a phase 1 study comparing evinacumab to placebo (in patients with TG levels between 150 and $500 \mathrm{mg} / \mathrm{dL}$ ) showed dosedependent reductions in TG levels up to $76.9 \%$ in the single ascending dose group and $83.1 \%$ in the multiple ascending dose group [95]. A phase 2 trial of evinacumb in patients with severe HTG (history of TG $>1000 \mathrm{mg} / \mathrm{dL}$ ) was completed in mid2020 , and results of the trial are eagerly anticipated.

\section{Conclusion}

Our understanding and management of HTG continue to evolve as new data emerges. Additional studies with more diverse patient demographics are needed to better understand the impact of genetics and environment on TG metabolism. Management strategies uniformly emphasize the importance of a healthy lifestyle, especially dietary attention and weight reduction when needed. TG lowering is critical to mitigate AP risk. Statin therapy is the foundation of pharmacotherapy for ASCVD risk reduction, and high-dose EPA has been shown to reduce risk in statin-treated adults with ASCVD or T2DM and moderate HTG. Novel therapies including nonselective PPAR-a, AngPTL3, and ApoC-III targeting may prove to be very important going forward to prevent and reduce cardiovascular disease and pancreatitis.

Acknowledgements It is noteworthy that this document was written during the global COVID-19 pandemic thanks to the contributions of a team of full-time clinicians and trainees whose efforts and time were being tested in extraordinary ways. Ms Hernandez has done an exceptional job bringing this all together. Thank you also to the invitation from Dr. Underberg to update this topic and for the cumulative lifetime contribution of Prof. Nodestgaard to inform this evolving topic.

\section{Declarations}

Conflict of Interest Dr. Soffer reports consultant for Akcea Therapeutics, Novartis; and investigator in clinical trials with Ionis, Amgen Inc., AstraZeneca, Ionis, Novartis, Pfizer, Regeneron, and REGENXBIO. Dr. Modarressi reports Speakers' Bureau for AstraZeneca. Dr. Bajaj reports investigator in clinical trials with Amgen Inc., Ionis, Novartis, Pfizer, Regeneron, and REGENXBIO. The other authors have nothing to disclose.

Human and Animal Rights and Informed Consent This article does not contain any studies with human or animal subjects performed by any of the authors.

\section{References}

Papers of particular interest, published recently, have been highlighted as:

- Of importance

$\bullet$ Of major importance

1. Nordestgaard BG. Triglyceride-rich lipoproteins and atherosclerotic cardiovascular disease: new insights from epidemiology, genetics, and biology. Circ Res. 2016;118:547-63.

2. Grundy SM, Stone NJ, Bailey AL, Beam C, Birtcher KK, Blumenthal RS, et al. 2018 AHA/ACC/AACVPR/AAPA/ABC/ ACPM/ADA/AGS/APhA/ASPC/NLA/PCNA guideline on the management of blood cholesterol: executive summary: a report of the American College of Cardiology/American Heart Association Task Force on Clinical Practice Guidelines. J Am Coll Cardiol. 2019;73:3168-209.

3. Mach F, Baigent C, Catapano AL, Koskinas KC, Casula M, Badimon L, et al. 2019 ESC/EAS guidelines for the management of dyslipidaemias: lipid modification to reduce cardiovascular risk. Eur Heart J. 2020;41:111-88.

4. Berglund L, Brunzell JD, Goldberg AC, Goldberg IJ, Sacks F, Murad MH, et al. Evaluation and treatment of hypertriglyceridemia: an endocrine society clinical practice guideline. J Clin Endocrinol Metab. 2012;97:2969-89.

5. Carroll M, Kit B, Lacher D. Trends in elevated triglyceride in adults: United States, 2001-2012. NCHS Data Brief. 2015;198.

6. Aguib Y, Al SJ. The Copenhagen City heart study (Østerbroundersøgelsen). Glob Cardiol Sci Pract. 2015;2015:33.

7. Esparza MI, Li X, Adams-Huet B, Vasandani C, Vora A, Das $\mathrm{SR}$, et al. Very severe hypertriglyceridemia in a large US county health care system: associated conditions and management. J Endocr Soc. 2019;3:1595-607.

8. DECODE Study Group, the European Diabetes Epidemiology Group. Glucose tolerance and cardiovascular mortality: comparison of fasting and 2-hour diagnostic criteria. Arch Intern Med. 2001;161:397-405.

9. Garg A. Lipodystrophies: genetic and acquired body fat disorders. J Clin Endocrinol Metab. 2011;96:3313-25.

10. Joseph J, Shamburek RD, Cochran EK, Gorden P, Brown RJ. Lipid regulation in lipodystrophy versus the obesity-associated metabolic syndrome: the dissociation of HDL-C and triglycerides. J Clin Endocrinol Metab. 2014;99:E1676-80.

11. Speliotes EK, Massaro JM, Hoffmann U, Vasan RS, Meigs JB, Sahani DV, et al. Fatty liver is associated with dyslipidemia and dysglycemia independent of visceral fat: the Framingham heart study. Hepatology. 2010;51:1979-87.

12. Smith GI, Shankaran M, Yoshino M, Schweitzer GG, Chondronikola M, Beals JW, et al. Insulin resistance drives hepatic de novo lipogenesis in nonalcoholic fatty liver disease. J Clin Investig. 2020;130:1453-60.

13. Petersen KF, Dufour S, Hariri A, Nelson-Williams C, Foo JN, Zhang X-M, et al. Apolipoprotein C3 gene variants in nonalcoholic fatty liver disease. N Engl J Med. 2010;362:1082-9.

14. Agrawal S, Zaritsky JJ, Fornoni A, Smoyer WE. Dyslipidaemia in nephrotic syndrome: mechanisms and treatment. Nat Rev Nephrol. 2018;14:57-70.

15. Clement LC, Macé C, Avila-Casado C, Joles JA, Kersten S, Chugh SS. Circulating angiopoietin-like 4 links proteinuria with hypertriglyceridemia in nephrotic syndrome. Nat Med. 2014;20:37-46.

16. Nikkilä EA, Kekki M. Plasma triglyceride metabolism in thyroid disease. J Clin Invest. 1972;51:2103-14. 
17. Duntas LH, Brenta G. The effect of thyroid disorders on lipid levels and metabolism. Med Clin North Am. 2012;96:269-81.

18. Yang L, Yin R, Wang Z, Wang X, Zhang Y, Zhao D. Circulating Angptl3 and Angptl8 are increased in patients with hypothyroidism. Biomed Res Int. 2019;2019:1-9.

19. Danese MD, Ladenson PW, Meinert CL, Powe NR. Effect of thyroxine therapy on serum lipoproteins in patients with mild thyroid failure: a quantitative review of the literature*. J Clin Endocrinol Metab. 2000;85:2993-3001.

20. Caraccio N, Ferrannini E, Monzani F. Lipoprotein profile in subclinical hypothyroidism: response to levothyroxine replacement, a randomized placebo-controlled study. J Clin Endocrinol Metab. 2002;87:1533-8.

21. Arnaldi G, Scandali VM, Trementino L, Cardinaletti M, Appolloni G, Boscaro M. Pathophysiology of dyslipidemia in Cushing's syndrome. Neuroendocrinology. 2010;92:86-90.

22. Macfarlane DP, Forbes S, Walker BR. Glucocorticoids and fatty acid metabolism in humans: fuelling fat redistribution in the metabolic syndrome. J Endocrinol. 2008;197:189-204.

23. Nikkilä EA, Pelkonen R. Serum lipids in acromegaly. Metabolism. 1975;24:829-38.

24. Murase T, Yamada N, Ohsawa N, Kosaka K, Morita S, Yoshida $\mathrm{S}$. Decline of postheparin plasma lipoprotein lipase in acromegalic patients. Metabolism. 1980;29:666-72.

25. Hansen I, Tsalikian E, Beaufrere B, Gerich J, Haymond M, Rizza $\mathrm{R}$. Insulin resistance in acromegaly: defects in both hepatic and extrahepatic insulin action. Am J Physiol Endocrinol Metab. 1986;250:E269-73.

26. Agledahl I, Skjærpe P-A, Hansen J-B, Svartberg J. Low serum testosterone in men is inversely associated with non-fasting serum triglycerides: the Troms $\varnothing$ study. Nutr Metab Cardiovasc Dis. 2008;18:256-62.

27. Chrysohoou C, Pitsavos D, Panagiotakos D, Oikonomou E, Lazaros G, Metaxa V, et al. Low testosterone levels are associated with metabolic syndrome, through triggering hypertriglyceridemia and low grade inflammation in elderly men. Ikaria study. Atherosclerosis. 2014;235:e155.

28. Wu FCW, Tajar A, Pye SR, Silman AJ, Finn JD, O'Neill TW, et al. Hypothalamic-pituitary-testicular axis disruptions in older men are differentially linked to age and modifiable risk factors: the european male aging study. J Clin Endocrinol Metab. 2008;93:2737-45

29. Pelkonen R, Nikkilä EA, Grahne B. Serum lipids, postheparin plasma lipase activities and glucose tolerance in patients with prolactinoma. Clin Endocrinol. 1982;16:383-90.

30. Oppenheim DS. Elevated serum lipids in hypogonadal men with and without hyperprolactinemia. Ann Intern Med. 1989;111:288.

31. Shibli-Rahhal A, Schlechte J. The effects of hyperprolactinemia on bone and fat. Pituitary. 2009;12:96-104.

32. Ling C, Svensson L, Odén B, Weijdegård B, Edén B, Edén S, et al. Identification of functional prolactin (PRL) receptor gene expression: PRL inhibits lipoprotein lipase activity in human white adipose tissue. J Clin Endocrinol Metab. 2003;88:1804-8.

33. Wild RA. Dyslipidemia in PCOS. Steroids. 2012;77:295-9.

34. Alexander CJ, Tangchitnob EP, Lepor NE. Polycystic ovary syndrome: a major unrecognized cardiovascular risk factor in women. Rev Obstet Gynecol. 2009;2:232-9.

35. Ghio A, Bertolotto A, Resi V, Volpe L, Di Cianni G. Triglyceride metabolism in pregnancy. Advances in Clinical Chemistry. Elsevier; 2011 [cited 2021 Apr 7]. p. 133-53. Available from: https://linkinghub.elsevier.com/retrieve/pii/B97801238704210 00071.

36. Knopp RH, Warth MR, Charles D, Childs M, Li JR, Mabuchi $\mathrm{H}$, et al. Lipoprotein metabolism in pregnancy, fat transport to the fetus, and the effects of diabetes. Neonatology. 1986;50:297-317.
37. Desoye G, Schweditsch MO, Pfeiffer KP, Zechner R, Kostner GM. Correlation of hormones with lipid and lipoprotein levels during normal pregnancy and postpartum*. J Clin Endocrinol Metab. 1987;64:704-12.

38 Barbour LA, McCurdy CE, Hernandez TL, Kirwan JP, Catalano PM, Friedman JE. Cellular mechanisms for insulin resistance in normal pregnancy and gestational diabetes. Diabetes Care. 2007;30:S112-9.

39. Goldberg AS, Hegele RA. Severe hypertriglyceridemia in pregnancy. J Clin Endocrinol Metab. 2012;97:2589-96.

40. Lewey J, Nemiroff R, Soffer D. Improving identification and treatment of primary and secondary hyperlipidemia for young women at a large health system. J Clin Lipidol. 2020;14:597.

41. Wong B, Ooi TC, Keely E. Severe gestational hypertriglyceridemia: a practical approach for clinicians. Obstet Med. 2015;8:158-67.

42. Barbour LA, Hernandez TL. Maternal non-glycemic contributors to fetal growth in obesity and gestational diabetes: spotlight on lipids. Curr Diab Rep. 2018;18:37.

43. Stone NJ. Secondary causes of hyperlipidemia. Med Clin North Am. 1994;78:117-41.

44. Glueck CJ, Fallat RW, Scheel D. Effects of estrogenic compounds on triglyceride kinetics. Metabolism. 1975;24:537-45.

45. Walsh BW, Schiff I, Rosner B, Greenberg L, Ravnikar V, Sacks FM. Effects of postmenopausal estrogen replacement on the concentrations and metabolism of plasma lipoproteins. N Engl J Med. 1991;325:1196-204.

46. Brown EE, Sturm AC, Cuchel M, Braun LT, Duell PB, Underberg JA, et al. Genetic testing in dyslipidemia: a scientific statement from the National Lipid Association. J Clin Lipidol. 2020;14:398-413. An important review of the uses of genetic testing for lipid disorders, and specific evidence based recommendations when there is HTG.

47. Moulin P, Dufour R, Averna M, Arca M, Cefalù AB, Noto $\mathrm{D}$, et al. Identification and diagnosis of patients with familial chylomicronaemia syndrome (FCS): expert panel recommendations and proposal of an "FCS score." Atherosclerosis. 2018;275:265-72.

48. Koopal C, Marais AD, Visseren FLJ. Familial dysbetalipoproteinemia: an underdiagnosed lipid disorder. Curr Opin Endocrinol Diabetes Obes. 2017;24:133-9.

49 Marais AD, Solomon GaE, Blom DJ. Dysbetalipoproteinaemia: a mixed hyperlipidaemia of remnant lipoproteins due to mutations in apolipoprotein E. Crit Rev Clin Lab Sci. 2014;51:46-62.

50. Sniderman AD, Thanassoulis G, Glavinovic T, Navar AM, Pencina M, Catapano A, et al. Apolipoprotein B particles and cardiovascular disease: a narrative review. JAMA Cardiol. 2019;4:1287.

51. Falko JM. Familial chylomicronemia syndrome: a clinical guide for endocrinologists. Endocr Pract. 2018;24:756-63.

52. D'Erasmo L, Di Costanzo A, Cassandra F, Minicocci I, Polito $\mathrm{L}$, Montali A, et al. Spectrum of mutations and long-term clinical outcomes in genetic chylomicronemia syndromes. ATVB. 2019;39:2531-41.

53. Brunzell JD, Albers JJ, Chait A, Grundy SM, Groszek E, McDonald GB. Plasma lipoproteins in familial combined hyperlipidemia and monogenic familial hypertriglyceridemia. J Lipid Res. 1983;24:147-55.

54. Gaddi A, Cicero AFG, Odoo FO, Poli AA, Paoletti R, Atherosclerosis and metabolic diseases study group. Practical guidelines for familial combined hyperlipidemia diagnosis: an update. Vasc Health Risk Manag. 2007;3:877-86.

55. Taghizadeh E, Esfehani RJ, Sahebkar A, Parizadeh SM, Rostami D, Mirinezhad M, et al. Familial combined hyperlipidemia: an overview of the underlying molecular mechanisms and therapeutic strategies. IUBMB Life. 2019;71:1221-9. 
56. Varbo A, Benn M, Tybjærg-Hansen A, Jørgensen AB, FrikkeSchmidt R, Nordestgaard BG. Remnant cholesterol as a causal risk factor for ischemic heart disease. J Am Coll Cardiol. 2013;61:427-36.

57. Do R, Willer CJ, Schmidt EM, Sengupta S, Gao C, Peloso GM, et al. Common variants associated with plasma triglycerides and risk for coronary artery disease. Nat Genet. 2013;45:1345-52.

58. Fei Y, Tsoi M-F, Cheung BMY. Cardiovascular outcomes in trials of new antidiabetic drug classes: a network meta-analysis. Cardiovasc Diabetol. 2019;18:112.

59. Arca M, Veronesi C, D’Erasmo L, Borghi C, Colivicchi F, De Ferrari GM, et al. Association of hypertriglyceridemia with allcause mortality and atherosclerotic cardiovascular events in a low-risk Italian population: the TG-REAL retrospective cohort analysis. JAHA. 2020 [cited 2021 Apr 7];9. Available from: https://www.ahajournals.org/doi/10.1161/JAHA.119.015801.

60. Murphy MJ, Sheng X, MacDonald TM, Wei L. Hypertriglyceridemia and acute pancreatitis. JAMA Intern Med. 2013;173:162.

61. Gaudet D, de Wal J, Tremblay K, Déry S, van Deventer S, Frei$\operatorname{dig} \mathrm{A}$, et al. Review of the clinical development of alipogene tiparvovec gene therapy for lipoprotein lipase deficiency. Atheroscler Suppl. 2010;11:55-60.

62. Yuan G, Al-Shali KZ, Hegele RA. Hypertriglyceridemia: its etiology, effects and treatment. Can Med Assoc J. 2007;176:1113-20.

63. Valdivielso P, Ramírez-Bueno A, Ewald N. Current knowledge of hypertriglyceridemic pancreatitis. Eur J Intern Med. 2014;25:689-94

64. Gonzales KM, Donato LJ, Shah P, Simha V. Measurement of apolipoprotein B levels helps in the identification of patients at risk for hypertriglyceridemic pancreatitis. J Clin Lipidol. 2021;15:97-103

65. Shenoy C, Shenoy M, Rao G. Dyslipidemia in dermatological disorders. North Am J Med Sci. 2015;7:421.

66. Vanessa Ngan, Staff Writer; Dr Amy Stanway, Dermatology Registrar, Nottingham, United Kingdom, 2005. Xanthoma. 2005. Available from: https://dermnetnz.org/topics/xanthoma/.

67. Davidson M, Stevenson M, Hsieh A, Ahmad Z, Crowson C, Witztum JL. The burden of familial chylomicronemia syndrome: interim results from the IN-FOCUS study. Expert Rev Cardiovasc Ther. 2017;15:415-23.

68. Miller M, Stone NJ, Ballantyne C, Bittner V, Criqui MH, Ginsberg HN, et al. Triglycerides and cardiovascular disease: a scientific statement from the American Heart Association. Circulation. 2011;123:2292-333.

69. Ford ES, Li C, Zhao G, Pearson WS, Mokdad AH. Hypertriglyceridemia and its pharmacologic treatment among US adults. Arch Intern Med. 2009;169:572.

70. Bays HE, Toth PP, Kris-Etherton PM, Abate N, Aronne LJ, Brown WV, et al. Obesity, adiposity, and dyslipidemia: a consensus statement from the National Lipid Association. J Clin Lipidol. 2013;7:304-83.

71. Jacobson TA, Maki KC, Orringer CE, Jones PH, Kris-Etherton $\mathrm{P}$, Sikand G, et al. National lipid association recommendations for patient-centered management of dyslipidemia: Part 2. J Clin Lipidol. 2015;9:1-S122.e1.

72. Mensink RP, Zock PL, Kester AD, Katan MB. Effects of dietary fatty acids and carbohydrates on the ratio of serum total to HDL cholesterol and on serum lipids and apolipoproteins: a meta-analysis of 60 controlled trials. Am J Clin Nutr. 2003;77:1146-55.

73. Johnson RK, Appel LJ, Brands M, Howard BV, Lefevre M, Lustig RH, et al. Dietary sugars intake and cardiovascular health: a scientific statement from the American Heart Association. Circulation. 2009;120:1011-20.

74. Kirkpatrick CF, Bolick JP, Kris-Etherton PM, Sikand G, Aspry $\mathrm{KE}$, Soffer DE, et al. Review of current evidence and clinical recommendations on the effects of low-carbohydrate and verylow-carbohydrate (including ketogenic) diets for the management of body weight and other cardiometabolic risk factors: a scientific statement from the National Lipid Association Nutrition and Lifestyle Task Force. J Clin Lipidol. 2019;13:689-711. e1.

75. Williams L, Rhodes KS, Karmally W, Welstead LA, Alexander L, Sutton L. Familial chylomicronemia syndrome: bringing to life dietary recommendations throughout the life span. J Clin Lipidol. 2018;12:908-19.

76. When Your Triglycerides Are Over $1,000 \mathrm{mg} / \mathrm{dL}$. https://www. lipid.org/. Available from: https://www.lipid.org/sites/default/ files/when_your_tgs_are_over_1000_mgdl.pdf.

77. Orringer CE, Jacobson TA, Maki KC. National lipid association scientific statement on the use of icosapent ethyl in statintreated patients with elevated triglycerides and high or very-high ASCVD risk. J Clin Lipidol. 2019;13:860-72.

78. Abdelhamid AS, Brown TJ, Brainard JS, Biswas P, Thorpe GC, Moore HJ, et al. Omega-3 fatty acids for the primary and secondary prevention of cardiovascular disease. Cochrane Database Syst Rev. 2018;11:CD003177.

79. Shearer GC, Savinova OV, Harris WS. Fish oil - how does it reduce plasma triglycerides? Biochim Biophys Acta (BBA) Mol Cell Biol Lipids. 2012;1821:843-51.

80. Skulas-Ray AC, Wilson PWF, Harris WS, Brinton EA, KrisEtherton PM, Richter CK, et al. Omega-3 fatty acids for the management of hypertriglyceridemia: a science advisory from the American Heart Association. Circulation. 2019 [cited 2021 Apr 11];140. Available from: https://www.ahajournals.org/doi/ 10.1161/CIR.0000000000000709.

81.• Bhatt DL, Steg PG, Miller M, Brinton EA, Jacobson TA, Ketchum SB, et al. Cardiovascular risk reduction with icosapent ethyl for hypertriglyceridemia. N Engl J Med. 2019;380:11-22. RCT demonstrating significant CVD risk reduction with high dose EPA (non-statin) added to optimal medical therapy for at-risk individuals with HTG.

82.•• Nicholls SJ, Lincoff AM, Garcia M, Bash D, Ballantyne CM, Barter PJ, et al. Effect of high-dose omega-3 fatty acids vs corn oil on major adverse cardiovascular events in patients at high cardiovascular risk: the STRENGTH randomized clinical trial. JAMA. 2020;324:2268. RCT failing to show benefit from high dose O3FA (EPA plus DHA) added to optimal medical therapy for at-risk individuals with HTG.

83. Staels B, Dallongeville J, Auwerx J, Schoonjans K, Leitersdorf E, Fruchart J-C. Mechanism of action of fibrates on lipid and lipoprotein metabolism. Circulation. 1998;98:2088-93.

84. Wiggins BS, Saseen JJ, Morris PB. Gemfibrozil in combination with statins - is it really contraindicated? Curr Atheroscler Rep. 2016;18:18.

85. Frick MH, Elo O, Haapa K, Heinonen OP, Heinsalmi P, Helo P, et al. Helsinki heart study: primary-prevention trial with gemfibrozil in middle-aged men with dyslipidemia. N Engl J Med. 1987;317:1237-45.

86. Rubins HB, Robins SJ, Collins D, Fye CL, Anderson JW, Elam $\mathrm{MB}$, et al. Gemfibrozil for the secondary prevention of coronary heart disease in men with low levels of high-density lipoprotein cholesterol. N Engl J Med. 1999;341:410-8.

87. Nordestgaard BG, Varbo A. Triglycerides and cardiovascular disease. The Lancet. 2014;384:626-35.

88. Arai H, Yamashita S, Yokote K, Araki E, Suganami H, Ishibashi S, et al. Efficacy and safety of K-877, a novel selective peroxisome proliferator-activated receptor $\alpha$ modulator (SPPARM $\alpha$ ), in combination with statin treatment: two randomised, doubleblind, placebo-controlled clinical trials in patients with dyslipidaemia. Atherosclerosis. 2017;261:144-52. 
89. Pradhan AD, Paynter NP, Everett BM, Glynn RJ, Amarenco $\mathrm{P}$, Elam M, et al. Rationale and design of the pemafibrate to reduce cardiovascular outcomes by reducing triglycerides in patients with diabetes (PROMINENT) study. Am Heart J. 2018;206:80-93.

90. Scott LJ. Alipogene tiparvovec: a review of its use in adults with familial lipoprotein lipase deficiency. Drugs. 2015;75:175-82.

91. Gaudet D, Stroes E, Bruno M, Andersen M, Petry H, Meyer C. Gene therapy with alipogene tiparvovec (glybera $\left.{ }^{\circledR}\right)$ for the prevention of LPLD induced pancreatitis: follow-up data suggests long-term clinical benefit. Atherosclerosis. 2014;235:e13.

92. Witztum JL, Gaudet D, Freedman SD, Alexander VJ, Digenio A, Williams KR, et al. Volanesorsen and triglyceride levels in familial chylomicronemia syndrome. N Engl $\mathbf{J}$ Med. 2019;381:531-42.

93. Gaudet D, Karwatowska-Prokopczuk E, Baum SJ, Hurh E, Kingsbury J, Bartlett VJ, et al. Vupanorsen, an N-acetyl galactosamine-conjugated antisense drug to ANGPTL3 mRNA, lowers triglycerides and atherogenic lipoproteins in patients with diabetes, hepatic steatosis, and hypertriglyceridaemia. Eur Heart J. 2020;41:3936-45.

94. A dose-ranging study with vupanorsen (TRANSLATE-TIMI 70). Clinicaltrials.gov. Available from: https://clinicaltrials.gov/ct2/ show/NCT04516291.

95. Ahmad Z, Banerjee P, Hamon S, Chan K-C, Bouzelmat A, Sasiela WJ, et al. Inhibition of angiopoietin-like protein 3 with a monoclonal antibody reduces triglycerides in hypertriglyceridemia. Circulation. 2019;140:470-86.

Publisher's Note Springer Nature remains neutral with regard to jurisdictional claims in published maps and institutional affiliations.

\section{Authors and Affiliations}

\section{Patricia Hernandez ${ }^{1}$. Neena Passi ${ }^{1} \cdot$ Taher Modarressi $^{2,3}$. Vivek Kulkarni ${ }^{1} \cdot$ Meshal Soni ${ }^{1} \cdot$ Fran Burke $^{1}$. Archna Bajaj ${ }^{1}$. Daniel Soffer ${ }^{1}$ (D)}

Patricia Hernandez

patricia.hernandez@pennmedicine.upenn.edu

Neena Passi

neena.passi@pennmedicine.upenn.edu

Taher Modarressi

modarressit@gmail.com

Vivek Kulkarni

vtkulkar@gmail.com

Meshal Soni

meshal.soni@pennmedicine.upenn.edu
Fran Burke

fburke@ pennmedicine.upenn.edu

Archna Bajaj

abajaj@pennmedicine.upenn.edu

Perelman School of Medicine, University of Pennsylvania, Philadelphia, PA, USA

2 Diabetes \& Endocrine Associates of Hunterdon, Flemington, NJ, USA

3 Hunterdon Medical Center, Flemington, NJ, USA 